\title{
GK Bootis and AE Fornacis: two low-mass eclipsing binaries with dwarf companions ${ }^{\star}, \star \star$
}

\author{
P. Zasche ${ }^{1}$, P. Svoboda ${ }^{2}$, and R. Uhlár ${ }^{3}$ \\ 1 Astronomical Institute, Faculty of Mathematics and Physics, Charles University Prague, CZ-180 00 Praha 8, V Holešovičkách 2, \\ Czech Republic \\ e-mail: zasche@sirrah.troja.mff.cuni.cz \\ 2 Private observatory, Výpustky 5, Brno 614 00, Czech Republic \\ 3 Private Observatory, Pohoří 71, 25401 Jílové u Prahy, Czech Republic
}

Received 6 June 2011 / Accepted 9 December 2011

\begin{abstract}
Context. A study of late-type low-mass eclipsing binaries provides us with important information about the most common stars in the Universe.

Aims. We obtain the first light curves and perform period analyses of two neglected eclipsing binaries GK Boo and AE For to reveal their basic physical properties.

Methods. We performed both a period analysis of the times of the minima and a $B V R$ light curve analysis. Many new times of minima for both the systems were derived and collected from the data obtained by automatic and robotic telescopes. This allowed us to study the long-term period changes in these systems for the first time. From the light curve analysis, we derived the first rough estimates of the physical properties of these systems.

Results. We find that the analyzed systems are somewhat similar to each other. Both contain low-mass components of similar types, both are close to the Sun, both have short orbital period, and both contain another low-mass companions on longer orbits of a few years. In the case of GK Boo, both components are probably of K3 spectral type, while the distant companion is probably a late M star. The light curve of GK Boo is asymmetric, which probably causes the shift in the secondary minima in the O-C diagram. System AE For comprises two K7 stars, and the third body is a possible brown dwarf with a minimal mass of only about $47 M_{\text {Jup }}$.

Conclusions. We succeed in completing period and light curve analyses of both systems, although a more detailed spectroscopic analysis is needed to confirm the physical parameters of the components to a higher accuracy.
\end{abstract}

Key words. binaries: eclipsing - stars: fundamental parameters - stars: individual: GK Boo - stars: individual: AE For

\section{Introduction}

Low-mass stars are the most common stars in our Galaxy (e.g. Kroupa 2002). However, owing to their low luminosity, only these close to the Sun have been studied in detail and many of them have never been analyzed. Hence, we focused on two rather neglected low-mass eclipsing binary systems: GK Boo and AE For. Their light curves as well as their period modulation had never been studied. Some studies indicate that most late-type stars are single (e.g. Lada 2006), but the number of papers studying the multiplicity of the late-type systems is still rather limited. Therefore, the incidence of multiples in late-type stars remains unexplored.

The study of eclipsing binaries provide us with important information about the physical properties of both of their components - their radii, masses, and evolutionary status. However, when considering only with the light curve, several assumptions have to be made. For the analysis presented in this paper we also used the photometric data obtained by automatic and robotic telescopes (such as ASAS, Pi of the sky, and SWASP). Thanks to these huge databases of observations, the long-term evolution of these systems can be studied for the first time.

\footnotetext{
* This paper uses observations made at the South African Astronomical Observatory (SAAO).

$\star \star$ Tables 8 and 9 are available in electronic form at http://www . aanda.org
}

\section{GK Boo}

\subsection{Introduction}

The system GK Boo (=BD+37 2556, $\left.V_{\max }=10.86 \mathrm{mag}\right)$ is an Algol-type eclipsing binary with an orbital period of about 0.48 day. It is also a primary component of a visual double designated WDS J14384+3632 in the Washington Double Star Catalog (WDS ${ }^{1}$, Mason et al. 2001). The secondary component of this double star is about 14" distant, and is probably gravitationally bound to GK Boo itself. It is about 0.4 mag fainter, but since its discovery in 1933 there has been no detectable mutual motion of the pair, hence the orbital period is of about thousands of years (rough estimation from the Kepler's law).

The star is too faint, thus was not observed by Hipparcos satellite, and its distance is therefore rather uncertain. Kharchenko (2001) introduced the parallax 30.29 mas, which is however only an estimate. Its spectral type is also unknown, but the $B-V$ index derived from the Tycho catalogue (Høg et al. 2000), $B-V=0.89$ mag indicates a spectral type of about K1. On the other hand, the 2MASS infrared photometry (Cutri et al. 2003) gives $J-H=0.527 \mathrm{mag}$ (therefore a spectral type of K3). Finally, Ammons et al. (2006) introduced a temperature corresponding to a spectral type of about K2-3. All these rough spectral estimates were taken from Popper (1980) and Cox (2000).

${ }^{1}$ http://ad.usno.navy.mil/wds/ 


\subsection{Light curve}

The star was observed by the SuperWASP (Pollacco et al. 2006) project and its complete light curve (hereafter LC) is available. However, we did not use these data for the LC analysis because these were not measured in any standard photometric filter. These data were only used to derive the minima times (see below). We observed the target at the Ondřejov observatory in the Czech Republic with the 65-cm telescope equipped with the CCD camera. For the light curve analysis, only the data from two nights in May 2011 were used (see Tables 8 and 9). The remaining observations were used for the minima time derivation and to analyze the period changes in the system (see below Sect. 2.3). The observations were obtained in standard $B, V$, and $R$ filters according to the specification of Bessell (1990).

At first, the complete LC was analyzed using the program PHoEbe (Prša \& Zwitter 2005), which is based on the WilsonDevinney algorithm (WD, Wilson \& Devinney 1971). The derived quantities are as follows: the secondary temperature $T_{2}$, the inclination $i$, the luminosities $L_{i}$, the gravity darkening coefficients $g_{i}$, the albedo coefficients $A_{i}$, and the synchronicity parameters $F_{i}$. The limb darkening was approximated using a linear law, and the values of $x_{i}$ were interpolated from the van Hamme's tables, given in van Hamme (1993).

At the beginning of the fitting process, we fixed the temperature of the primary component at $T_{1}=4700 \mathrm{~K}$ (corresponding to spectral type K3, Cox 2000). In the absence of spectroscopy, the mass ratio was derived via a so-called "q-search method". This means that we tried different values of mass ratio in the range $1.5-0.5$ in steps of 0.1 and tried to find the best LC fit according to the lowest value of rms. Finally, we found that the best-fit solution was reached with the value $q=M_{2} / M_{1}=0.9$, which agrees with both eclipses having almost equal depths. For a given mass ratio, the semi-major axis was fixed to an appropriate value for the primary mass to be equal to a typical mass of a particular spectral type (e.g. Popper 1980; Harmanec 1988; or Andersen 1991). With this approach, we were able to estimate the masses, in addition to the radii of both components in absolute units.

However, during the LC fitting process we found that the LC of GK Boo is asymmetric. In particular, the part of the LC near the secondary minimum is distorted in all $B V R$ filters. The brightness just after the ascent from the secondary minimum (near the phase 0.6) is higher than the brightness just before the descent (phase 0.4). The difference is about $0.022 \mathrm{mag}$ in $B, 0.018 \mathrm{mag}$ in $V$, and $0.017 \mathrm{mag}$ in $R$ filter, respectively.

With the PHOEBE code, we tried to fix the values of $A_{i}$ and $g_{i}$ to their appropriate values of 0.5 and 0.32 , respectively. However, after then we also allowed these parameters to be fitted, because the fit is tighter (rms). However, probably owing to the asymmetry of the LC these quantities converged to the rather improbable values given in Table 1, and the shape of the observed LC could not be fitted properly. For the asymmetry of the curve, we also tried to introduce a star spot on either of the components. However, no acceptable solution with spot(s) was found to describe the shape of the light curve more accurately in the PHOEBE program. The parameters of the LC fit are given in Table 1, but these cannot sufficiently describe the shape of the LC.

We therefore tried a different code, called RocHE, developed by Theo Pribulla (Pribulla 2004), which is also based on the WD code but has for instance also some other computing methods and different controlling of the calculation process. With this program, we used two star spots and similar input parameters as described above. At the beginning, the values of $A_{i}$ and $g_{i}$ were fixed to the appropriate values of 0.5 and 0.32 , respec-
Table 1. Light curve parameters of GK Boo.

\begin{tabular}{lcc}
\hline \hline Parameter & \multicolumn{2}{c}{ Value } \\
& PHOEBE & ROCHE \\
\hline$T_{1}[\mathrm{~K}]$ & \multicolumn{2}{c}{$4700^{*}$} \\
$T_{2}[\mathrm{~K}]$ & $4540 \pm 50$ & $4615 \pm 63$ \\
$q\left(=M_{2} / M_{1}\right)$ & $0.9 \pm 0.1$ & $0.95 \pm 0.12$ \\
$e$ & \multicolumn{2}{c}{$0^{*}$} \\
$i$ [deg] & $89.83 \pm 0.57$ & $89.28 \pm 0.37$ \\
$g_{1}$ & $0.00 \pm 0.04$ & $0.35 \pm 0.05$ \\
$g_{2}$ & $0.00 \pm 0.03$ & $0.35 \pm 0.05$ \\
$A_{1}$ & $0.00 \pm 0.08$ & $0.80 \pm 0.05$ \\
$A_{2}$ & $1.00 \pm 0.08$ & $0.80 \pm 0.05$ \\
$F_{1}$ & $1.892 \pm 0.107$ & $1.131 \pm 0.096$ \\
$F_{2}$ & $1.866 \pm 0.116$ & $1.295 \pm 0.108$ \\
$L_{1}(B)[\%]$ & $54.8 \pm 1.9$ & $52.4 \pm 1.1$ \\
$L_{2}(B)[\%]$ & $45.2 \pm 1.8$ & $47.6 \pm 1.0$ \\
$L_{1}(V)[\%]$ & $53.5 \pm 1.5$ & $51.6 \pm 1.2$ \\
$L_{2}(V)[\%]$ & $46.5 \pm 1.3$ & $48.4 \pm 1.1$ \\
$L_{1}(R)[\%]$ & $52.1 \pm 1.4$ & $51.1 \pm 1.1$ \\
$L_{2}(R)[\%]$ & $47.9 \pm 1.3$ & $48.9 \pm 1.0$ \\
$l_{1}[\mathrm{deg}]$ & Spots: & \\
$b_{1}[\mathrm{deg}]$ & - & $287.2 \pm 7.9$ \\
$r_{1}[\mathrm{deg}]$ & - & $60.5 \pm 3.2$ \\
$k_{1}$ & - & $37.9 \pm 2.0$ \\
$l_{2}[\mathrm{deg}]$ & - & $0.75 \pm 0.04$ \\
$b_{2}[\mathrm{deg}]$ & - & $63.3 \pm 7.4$ \\
$r_{2}[\mathrm{deg}]$ & - & $47.4 \pm 12.8$ \\
$k_{2}$ & - & $28.7 \pm 4.1$ \\
\hline$R_{1}\left[R_{\odot}\right]$ & - & $0.76 \pm 0.04$ \\
$R_{2}\left[R_{\odot}\right]$ & $0.83 \pm 0.18$ \\
$M_{1}\left[M_{\odot}\right]$ & $0.86 \pm 0.18$ & $0.89 \pm 0.15$ \\
$M_{1}\left[M_{\odot}\right]$ & $0.66 \pm 0.06$ & $0.86 \pm 0.14$ \\
\hline & \multicolumn{2}{c}{$0.73 \pm 0.06$} \\
\hline
\end{tabular}

Notes. ${ }^{(*)}$ Fixed.

tively. However, to achieve a tighter fit both $A_{i}$ and $g_{i}$ values were also varied across the range from 0 to 1 in steps of 0.05 for both components. The synchronicity parameters $F_{i}$ converged to much more reliable values. The value of mass ratio was fixed to $q=1.0$ and then also fitted as a free parameter. This was possible because there is a clear distortion of the LC outside the minima (see e.g. Terrell \& Wilson 2005). For the fitting process, the two different limb darkening laws were also tried, namely a linear and logarithmic. The latter one provides a much tighter fit to our data. All of the resulting LC parameters are also given in Table 1 (together with parameters of two cooler spots located on the primary component - longitude, latitude, radius and temperature factor). As one can see, the two solutions clearly differ even outside their respective error bars for some of the parameters.

The individual errors in the parameters were not taken from the WD code, but derived in the following way. We computed a range of solutions for GK Boo, which were then used for its error estimation. All solutions with $\chi^{2}$ value close to the minimal one (5\% from our final solution) were taken and the resultant values of parameters were used to compute the differences between the parameters. The errors in the individual parameters were then computed as a maximum difference and their individual WD errors, given by $\max \left(a_{i}-a_{\min }\right)+\delta a_{i}+\delta a_{\min }$.

This solution obtained with the ROCHE program provides a much closer fit to the observed data and is the fit plotted in Fig. 1. The value of the eccentricity was fixed at 0 (for a discussion about possible eccentricity see below). Our resultant parameters indicate that both the components are still located on 
P. Zasche et al.: GK Boo and AE For: Two low-mass eclipsing binaries with dwarf companions

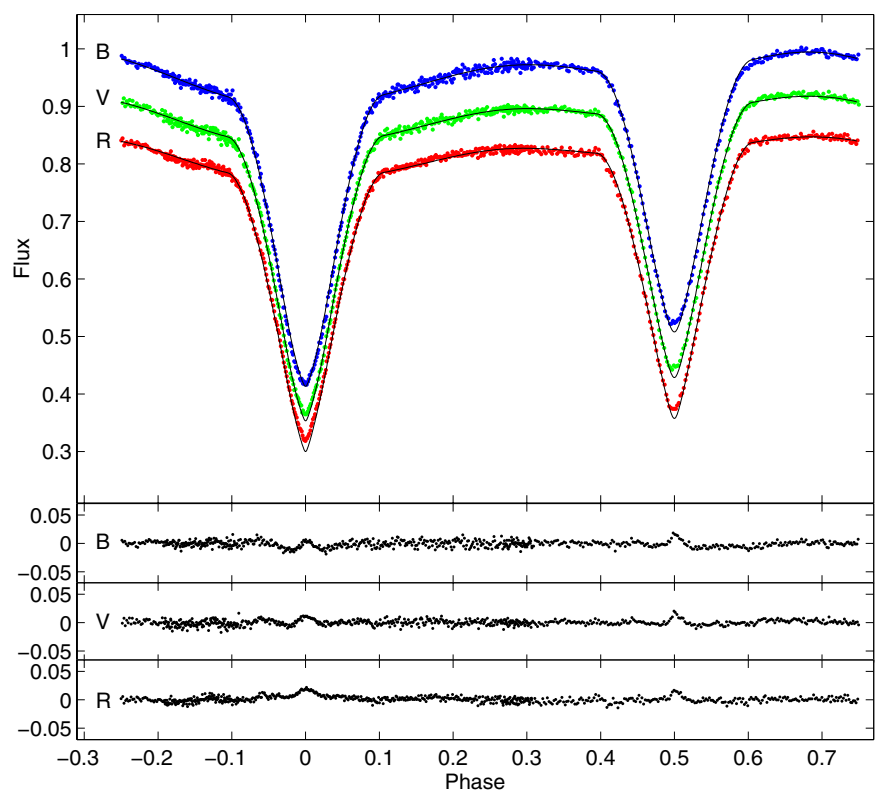

Fig. 1. Light curves in $B V R$ filters for GK Boo, the solid lines represent the final fit. The residuals after the fit are plotted below. The curves are shifted along $y$-axis for reasons of clarity.

the main sequence, (as required because the age of the Universe does not allow low-mass stars to have evolved from the main sequence). If we follow the assumption of a $\mathrm{K} 3 \mathrm{~V}$ primary, then the secondary is also of $\mathrm{K} 3 \mathrm{~V}$ spectral type. These are consistent with the photometric indices presented above, as well as with the individual masses and radii for these types of stars (e.g. Harmanec 1988). An undetectable value of the third light was also resulted derived by this analysis. The presence of photospheric spots on both components of such a late spectral type star is also foreseeable.

\subsection{Period analysis}

To monitor the detailed long-term evolution of the system or its short-period modulation, we collected all available published minima observations. Photometry from the SWASP (Pollacco et al. 2006), ASAS (Pojmanski 2002), and PiOfTheSky (Burd et al. 2005) projects were used to derive many new minima times for GK Boo. All of these data are given in Table 8. The method of Kwee \& van Woerden (1956) was used. Some of the data were of poor quality, but most were accurate enough to perform a detailed period analysis of the system. The range of these data is about 12 years.

We used these data to analyze the period modulation and found some interesting results. Applying the hypothesis of a third body in the system (the so-called LIght-Time Effect, hereafter LITE, described e.g. by Irwin 1959), we found a weak period modulation with a period of about four years. The final fit to the data together with the theoretical curve is shown in Fig. 2. As one can see, there is also some long-term period evolution of the orbital period (the blue dashed line), which was described as a quadratic term in ephemerides. It can be understood as a slow period decrease caused by the mass loss from the system or mass flow between the components (or even momentum loss, magnetic breaking, etc.). Another explanation is that this is only part of the long-term period modulation, although we have only limited data coverage.

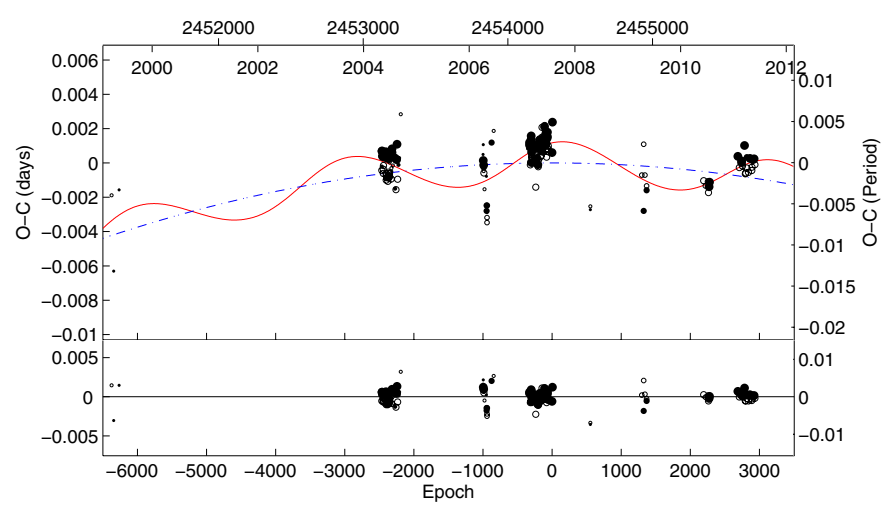

Fig. 2. Periodic modulation of period GK Boo. Blue dashed line represents quadratic ephemeris, while red solid line stands for the LITE hypothesis. Residuals are plotted in the bottom plot. The larger the symbol, the higher the weight (higher the precision).

Table 2. Final parameters of the long orbit for GK Boo.

\begin{tabular}{lc}
\hline \hline Parameter & Value \\
\hline HJD $_{0}$ & $2454305.4570 \pm 0.0006$ \\
$P$ [day] & $0.47777174 \pm 0.00000022$ \\
$p_{3}[$ day $]$ & $1472.7 \pm 170.0$ \\
$p_{3}$ [yr] & $4.032 \pm 0.450$ \\
$A$ [day] & $0.0126 \pm 0.0012$ \\
$T_{0}$ & $2454263.3 \pm 1108.3$ \\
$\omega_{3}[$ deg $]$ & $56.54 \pm 15.0$ \\
$e_{3}$ & $0.084 \pm 0.267$ \\
$Q\left[\times 10^{-10}\right]$ & $-1.071 \pm 0.206$ \\
\hline$f\left(M_{3}\right)\left[M_{\odot}\right]$ & $0.000633 \pm 0.000002$ \\
$M_{3, \min }\left[M_{\odot}\right]$ & $0.115 \pm 0.001$ \\
$M_{3,60}\left[M_{\odot}\right]$ & $0.134 \pm 0.002$ \\
$M_{3,30}\left[M_{\odot}\right]$ & $0.242 \pm 0.005$ \\
$a_{12} \sin i[\mathrm{AU}]$ & $0.217 \pm 0.108$ \\
$a_{3}[\mathrm{mas}]$ & $88.7 \pm 9.8$ \\
\hline
\end{tabular}

A more interesting finding is that of a period of about 4 years. Applying the LITE hypothesis, we obtained a final set of parameters given in Table 2, namely the period of the third body $p_{3}$, the semi-amplitude of the effect $A$, the time of periastron passage $T_{0}$, the argument of periastron $\omega_{3}$, and the eccentricity $e_{3}$. Despite the low amplitude (about only $1.8 \mathrm{~min}$ ) of the LITE, most of the observed minima times are of higher precision and the modulation is clearly visible. Table 2 also provides the mass function of the third body $f\left(M_{3}\right)$, which helps us to estimate its predicted mass.

Having no information about the inclination between the orbits of the eclipsing pair and the hypothetical third body, we plotted Fig. 3, where a plot mass versus inclination is shown. Assuming the coplanar orbits (i.e. $i_{3}=90^{\circ} \rightarrow M_{3}=M_{3, \min }$ ), the resulted minimum mass of the third body is only about $0.116 M_{\odot}$, which places this body at the lower end of stellar masses, hence we can rule out the hypothesis of a brown dwarf or even an exoplanet. Despite of there being no upper limit to this mass (it goes to infinity with $i_{3} \rightarrow 0^{\circ}$ ), we can estimate a lower limit to the mass. Taking into account that no third light is detected in the LC solution, e.g. $L_{3} /\left(L_{1}+L_{2}\right)<0.01$ and assuming a main-sequence star, we can estimate its mass to be lower than $0.22 M_{\odot}$, which is shown in Fig. 3 as a gray area. Further observations are still needed to confirm this hypothesis with higher conclusiveness.

If we assume the parallax of GK Boo as given by Kharchenko (2001), $\pi=30.29$ mas, we are also able to compute 


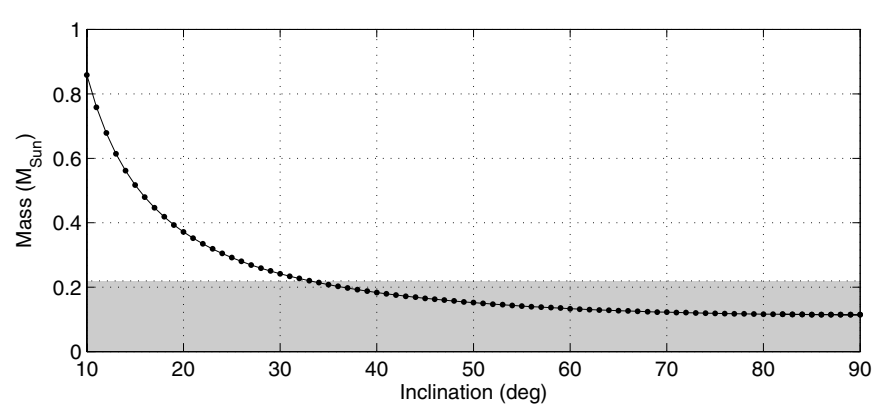

Fig. 3. GK Boo: mass of the third body based on from the LITE hypothesis with respect to the inclination between the orbits.

Table 3. Apsidal motion parameters for GK Boo.

\begin{tabular}{lc}
\hline \hline Parameter & Value \\
\hline$e$ & $0.0944 \pm 0.0068$ \\
$\omega[\mathrm{deg}]$ & $268.9 \pm 2.5$ \\
$\dot{\omega}[\mathrm{deg} /$ cycle $]$ & $0.00026 \pm 0.00001$ \\
$U[\mathrm{yr}]$ & $1790 \pm 50$ \\
\hline
\end{tabular}

the angular distance of a hypothetical body to be about 89 mas. This separation of components is well above the limit for modern stellar interferometers. However, there is a problem with the brightness of the third component, which was found to be about more than five magnitudes fainter than the eclipsing pair itself. With the brightness of about 11 mag for the system, this makes a detection impossible. The magnitude difference of the third body with respect to the close pair also clarify why no third light was detected in the LC solution.

Another interesting result was a detection of displaced secondaries. This can be clearly seen in more precise data points (SWASP and our new observations). That secondary minima occur at a different phase of $\phi_{2} \neq 0.5$ from the primary usually indicates that the system is on an eccentric orbit. GK Boo is a well-detached system, so the eccentric orbit cannot be ruled-out easily. Therefore, we assumed an apsidal motion hypothesis for our data set of minima times. We followed a procedure described by e.g. Giménez \& García-Pelayo (1983) or Giménez \& Bastero (1995) and obtained a set of apsidal motion parameters. The plot of residuals (after subtraction of the LITE fit) with the apsidal motion fit is shown in Fig. 4. It is obviously very slow because the position of secondaries versus primaries changes only very slowly. The resultant values of apsidal motion parameters are given in Table 3.

However, we have to rule out this hypothesis because it lead to unacceptable results. With some information about the physical parameters of both components, we can use the apsidal motion parameters to estimate the internal structure constant. The theoretical $\log k_{2, \text { theor }}$ value taken from Claret (2004) should range from -1.35 to -1.65 . However, the mean value of $\log k_{2}$ of both components that can be derived from our solution is very different, even when $k_{2}<0$, which is unacceptable. Thus, the system is very probably on a circular orbit.

We may ask why the secondary minima deviate from the 0.5 phase. We published a finding that the displaced secondary minima can also be present in contact binaries where no eccentric orbit is possible (Zasche 2011), so one cannot perform an apsidal motion analysis based only on the minima times of a particular system. Some studies found that the secondary minimum is displaced because of the distortion of the LC, thus any standard routine for deriving the time of minimum (e.g. Kwee-van Woerden,

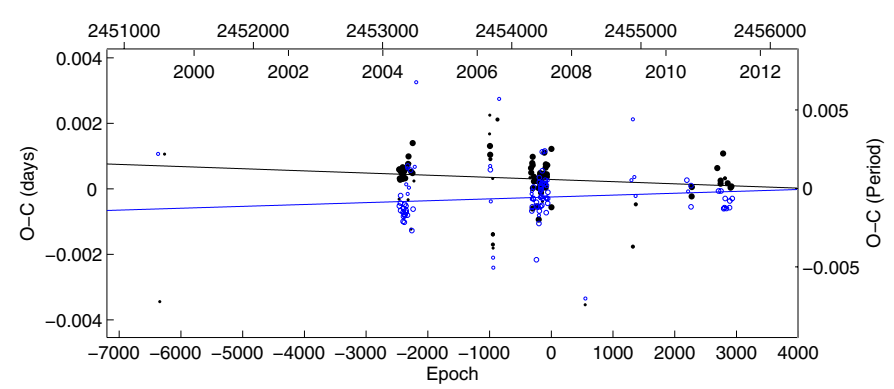

Fig. 4. O-C diagram of GK Boo with the apsidal motion hypothesis, black color is for primary minima, while blue one for secondary.

Table 4. Methods of minima fitting for GK Boo.

\begin{tabular}{lcc}
\hline \hline Method of minima fitting & rms & BIC \\
\hline Linear ephemeris: & 0.00151 & 23.5 \\
Quadratic ephemeris: & 0.00119 & 29.2 \\
LITE and linear ephemeris: & 0.00080 & 51.0 \\
LITE and quadratic ephemeris: & 0.00074 & 56.4 \\
LITE, quadratic ephemeris and apsidal motion: & 0.00060 & 72.6 \\
\hline
\end{tabular}

bisector chord method or polynomial fitting) cannot be used properly because these consider symmetric minima only. When using these methods to determine minima where both ascending and descending branches have different slopes, we recover only a "false eccentricity".

One can also ask about a significance of the fits presented in Figs. 2 and 4. For this comparison, we summarized different approaches in Table 4. In addition to the rms values, we also provide the values of BIC (Bayesian Information Criterion, see e.g. Liddle 2007), which show the significance of the fit. According to this method, the smaller the rms value, the tighter the fit. To conclude, our final fit provides the smallest rms, but its significance is low and still highly speculative. This is also caused by the poor data coverage, and large scatter in the minima and their low accuracy. Determinations of new more precise minima are therefore needed to confirm or exclude this hypothesis.

\section{AE For}

\subsection{Introduction}

The Algol-type system AE For (=HIP 14568, $V_{\max }=$ $10.22 \mathrm{mag}$ ) is also a poorly studied binary. Its published spectral types range from $\mathrm{K} 4$ to $\mathrm{M} 0$, with the most probable one being K7V as derived by (Torres et al. 2006). The system was presented as a wide double with the star HD 19632 based on their similar parallaxes and proper motions (see Poveda et al. 1994).

Neither the light curve nor the radial velocity curve of AE For have been studied. The star was observed by the Hipparcos satellite and a few times also for the minima observations. It was also continuously monitored with automatic photometric systems such as PiOfTheSky and ASAS. However, the quality of these data do not allow us to use them for a LC analysis. The distance to the system was derived from the Hipparcos data to be $d=31.5 \mathrm{pc}$.

\subsection{Light curve}

We observed the star from the South African Astronomical Observatory (SAAO) in 2010, using the classical one-channel photoelectric photometer mounted on the $50-\mathrm{cm}$ telescope. All 
P. Zasche et al.: GK Boo and AE For: Two low-mass eclipsing binaries with dwarf companions

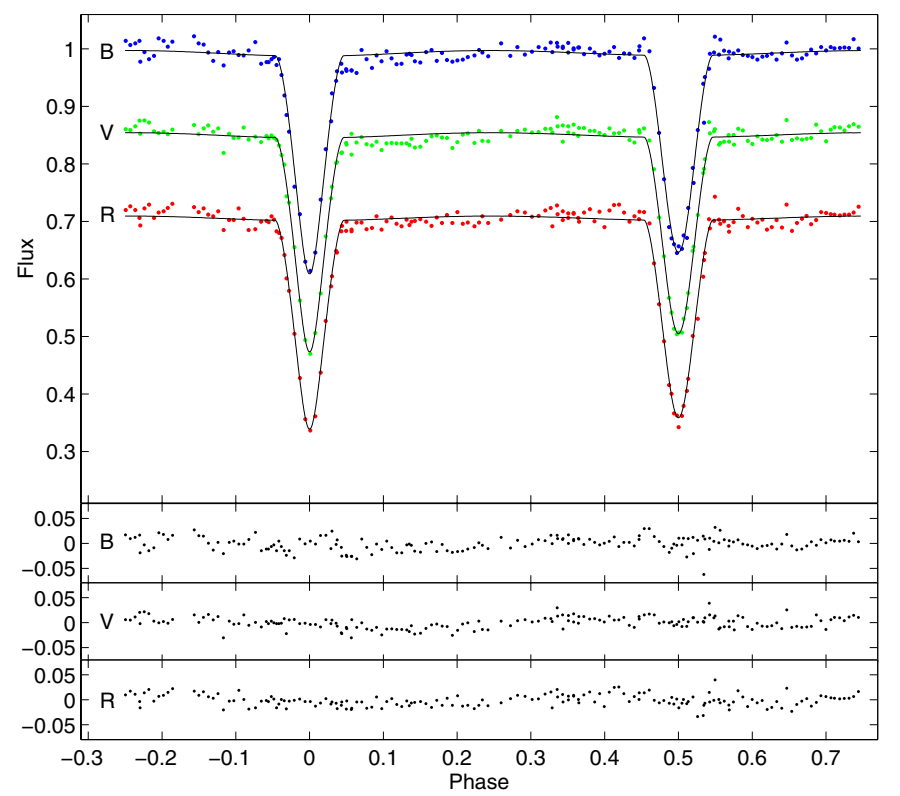

Fig. 5. Light curve in $B V R$ filters for AE For, the solid line represents the final fit. The curves are shifted along the y-axis for greater clarity.

measurements were carefully reduced to the Cousins E-region standard system (Menzies et al. 1989) and corrected for differential extinction.

Thanks to its orbital period close to one day, its complete light curve was observed once in standard $B V R$ filters, with some overlapping points (about 170 data points in each filter were obtained). Unfortunately, the quality of the data acquired for several nights was not very good, hence the scatter in the curve is affected by these conditions. Two secondary and one primary minima were observed (see below).

We analyzed our data using the same computational procedure as for GK Boo. The primary temperature was fixed to the

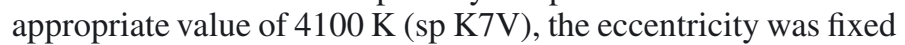
to 0 , the values of gravity darkening coefficients were fixed at 0.32 , and the albedo coefficients to 0.5 (as recommended for stars with convective envelopes), while the limb darkening coefficients were interpolated from values given in van Hamme (1993). The computational approach was different for the mass ratio $q$, which was fixed to $q=1.0$ because of the weak outsideeclipse ellipsoidal variations and its detached configuration. In addition, the synchronicity parameters $F_{i}$ were set to values of 1.0 for both components. The program ROCHE was used and the resulting LC parameters are given in Table 5, while the final solution is presented in Fig. 5.

One can see that the secondary temperature $T_{2}$ is close to the value of $T_{1}$, indicating that the components are similar. Thus, the estimated spectral types of both stars are probably K7V + K7V. Both components are still located on the main sequence and their properties are in agreement with the typical values of K7V stars (as presented by e.g. Harmanec 1988). The third light was also not detected here in any filter. In contrast to GK Boo, the LC of AE For seems to be symmetric.

\subsection{Period analysis}

Similarly to GK Boo, we tried to perform the period analysis of all available minima. The collection of minima is much smaller, but thanks to the first observation by Hipparcos (Perryman et al. 1997) these cover a longer time span than for GK Boo. Several
Table 5. Light curve parameters of AE For.

\begin{tabular}{lc}
\hline \hline Parameter & Value \\
\hline$T_{1}[\mathrm{~K}]$ & $4100^{*}$ \\
$T_{2}[\mathrm{~K}]$ & $4065 \pm 48$ \\
$q\left(=M_{2} / M_{1}\right)$ & $1.0^{*}$ \\
$e$ & $0^{*}$ \\
$i[\mathrm{deg}]$ & $86.51 \pm 0.31$ \\
$g_{1}=g_{2}$ & $0.32^{*}$ \\
$A_{1}=A_{2}$ & $0.50^{*}$ \\
$F_{1}=F_{2}$ & $1.000^{*}$ \\
$L_{1}(B)[\%]$ & $63.2 \pm 1.3$ \\
$L_{2}(B)[\%]$ & $36.8 \pm 1.0$ \\
$L_{1}(V)[\%]$ & $63.1 \pm 1.2$ \\
$L_{2}(V)[\%]$ & $36.9 \pm 1.0$ \\
$L_{1}(R)[\%]$ & $62.6 \pm 1.4$ \\
$L_{2}(R)[\%]$ & $37.4 \pm 1.0$ \\
\hline \multicolumn{2}{c}{ Derived quantities: } \\
$R_{1}\left[R_{\odot}\right]$ & $0.66 \pm 0.10$ \\
$R_{2}\left[R_{\odot}\right]$ & $0.52 \pm 0.08$ \\
$M_{1}\left[M_{\odot}\right]$ & $0.50 \pm 0.05$ \\
$M_{1}\left[M_{\odot}\right]$ & $0.50 \pm 0.05$ \\
\hline \multicolumn{2}{c}{}
\end{tabular}

Notes. ${ }^{(*)}$ Fixed.
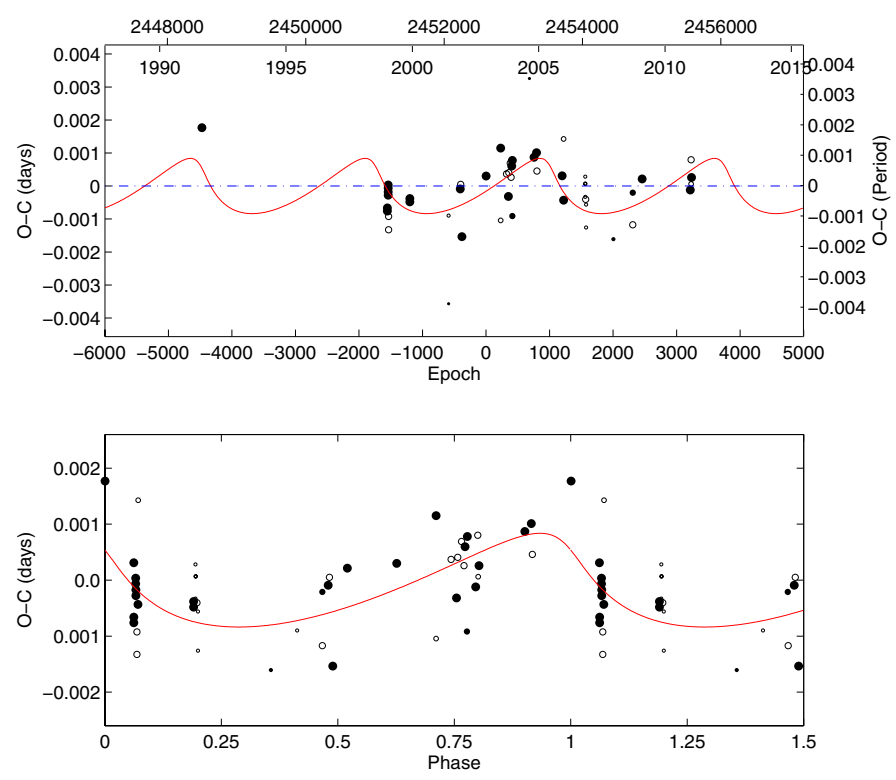

Fig. 6. O-C diagram of AE For. up: with linear ephemeris. Bottom: with respect to the phase. The data points are fitted with the curve representing the third body hypothesis (see the text for details).

new minima were derived based on our new observations from SAAO as well as those from the ASAS and PiOfTheSky surveys.

The same hypothesis as for GK Boo was applied to the data points here. All of the minima times used for the analysis are summarized in Table 9. As one can see from Fig. 6, there is a clear variation in the minima times. We used the same third-body hypothesis (LITE) as for GK Boo, deriving a final fit to the data given by the parameters in Table 6 . The LITE hypothesis resulted in a rather eccentric orbit, although the result is affected by a relatively large error, hence maybe the $e_{3}$ value should be lower. Only additional observations would help us confirm or refute this hypothesis, refine the period, and possibly detect some longterm evolution of the period similar to that in GK Boo, because the first observation from Hipparcos deviates significantly from the fit. With the same procedure as for GK Boo, we computed the significance of the fits according to the BIC criterion (see Table 
Table 6. Final parameters of the long orbit for AE For.

\begin{tabular}{lc}
\hline \hline Parameter & Value \\
\hline $\mathrm{HJD}_{0}$ & $2452605.97070 \pm 0.00035$ \\
$P$ [day] & $0.91820943 \pm 0.00000012$ \\
$p_{3}$ [day] & $2524.6 \pm 149.6$ \\
$p_{3}$ [yr] & $6.912 \pm 0.409$ \\
$A$ [day] & $0.00083 \pm 0.00032$ \\
$T_{0}$ & $2453548.8 \pm 413.1$ \\
$\omega_{3}[\mathrm{deg}]$ & $146.2 \pm 57.8$ \\
$e_{3}$ & $0.601 \pm 0.414$ \\
\hline$f\left(M_{3}\right)\left[M_{\odot}\right]$ & $0.000098 \pm 0.000001$ \\
$M_{3, \min }\left[M_{\odot}\right]$ & $0.047 \pm 0.001$ \\
$M_{3,60}\left[M_{\odot}\right]$ & $0.055 \pm 0.001$ \\
$M_{3,30}\left[M_{\odot}\right]$ & $0.098 \pm 0.003$ \\
$a_{12} \sin i[\mathrm{AU}]$ & $0.167 \pm 0.064$ \\
$a_{3}[\mathrm{mas}]$ & $117.2 \pm 8.3$ \\
\hline
\end{tabular}

Table 7. Methods of minima fitting for AE For.

\begin{tabular}{lcc}
\hline \hline Method of minima fitting & rms & BIC \\
\hline Linear ephemeris: & 0.000255 & 24.4 \\
LITE and linear ephemeris: & 0.000163 & 44.8 \\
\hline
\end{tabular}

7). As one can see, the fit is still very poor and highly speculative. However, using only the linear ephemeris, there remains a clear quasi-sinusoidal variation, which needs some physical explanation.

From the LITE parameters, we were able to calculate the minimal mass of the third body (i.e. coplanar orbits), which we found to be only about $47 M_{\text {Jup }}$, which is even lower than the limit of stellar masses. Therefore, if the orbits were coplanar (which only would be our assumption, because the process of tidal coplanarization is very slow), the third body would very probably be a brown dwarf (exoplanets have masses about one half of this value). With such a body, we reach minimal masses that can be detected by this method, because the amplitude of LITE is comparable to the typical precision of individual minima-time measurements. Whatever applies to the possible interferometric detection of GK Boo companion also applies here, because its luminosity is too low.

\section{Discussion and conclusions}

We have derived preliminary light-curve solutions and period analyses of the poorly studied Algol-type eclipsing binaries GK Boo and AE For, which we have found to have several interesting and similar features. Since both of them are low-mass stars of very similar types $(\mathrm{K} 3+\mathrm{K} 3$ for $\mathrm{GK}$ Boo, and $\mathrm{K} 7+\mathrm{K} 7$ for AE For), both of them have short orbital periods. Moreover, both are relatively close to the Sun and also appear to contain third bodies in their systems, which cause a periodic modulation of the orbital periods of both systems. Assuming a coplanar orbit, for AE For this third body appears to be a brown dwarf, which makes this system even more interesting. However, more photometric and spectroscopic observations are needed to confirm or refute this hypothesis.

The system GK Boo has an asymmetric light curve, which is the probably accounts for the shift in the secondary minimum in phase with the primary one. The apsidal motion hypothesis cannot explain this discrepancy.

In general, if the third body hypothesis as proposed based on the period analysis is found to be the correct one, here we have considered quite curious examples of hierarchical quadruple systems of low masses. As far as we know, there are only a few similar multiple late-type systems for which one of the components is an eclipsing binary (e.g. BB Scl or MR Del).

Acknowledgements. We thank the "ASAS", "SWASP" and "Pi of the sky" teams for making all of the observations easily public available. P.Z. wish to thank the staff at SAAO for their warm hospitality and help with the equipment. Authors are also grateful to Theo Pribulla for his helpful comments about the ROCHE code. An anonymous referee is also acknowledged for his/her helpful and critical suggestions. This work was supported by the Czech Science Foundation grant No. P209/10/0715 and also by the Research Programme MSM0021620860 of the Czech Ministry of Education. This research has made use of the SIMBAD database, operated at CDS, Strasbourg, France, and of NASA's Astrophysics Data System Bibliographic Services.

\section{References}

Ammons, S. M., Robinson, S. E., Strader, J., et al. 2006, ApJ, 638, 1004 Andersen, J. 1991, A\&ARv, 3, 91

Bessell, M. S. 1990, PASP, 102, 1181

Burd, A., Cwiok, M., Czyrkowski, H., et al. 2005, NewA, 10, 409

Claret, A. 2004, A\&A, 424, 919

Cox, A. N. 2000, Allen's Astrophysical Quantities

Cutri, R. M., Skrutskie, M. F., van Dyk, S., et al. 2003, VizieR Online Data Catalog, 2246, 0

Giménez, A., \& Bastero, M. 1995, Ap\&SS, 226, 99

Giménez, A., \& García-Pelayo, J. M. 1983, Ap\&SS, 92, 203

van Hamme, W. 1993, AJ, 106, 2096

Harmanec, P. 1988, BAICz, 39, 329

Høg, E., Fabricius, C., Makarov, V. V., et al. 2000, A\&A, 355, L27

Irwin, J. B. 1959, AJ, 64, 149

Kharchenko, N. V. 2001, Kinematika i Fizika Nebesnykh Tel, 17, 409

Kroupa, P. 2002, Science, 295, 82

Kwee, K. K., \& van Woerden, H. 1956, BAN, 12, 327

Lada, C. J. 2006, ApJ, 640, L63

Liddle, A. R. 2007, MNRAS, 377, L74

Mason, B. D., Wycoff, G. L., Hartkopf, W. I., Douglass, G. G., \& Worley, C. E. 2001, AJ, 122, 3466

Menzies, J. W., Cousins, A. W. J., Banfield, R. M., \& Laing, J. D. 1989, SAAOC, 13,1

Perryman, M. A. C., Lindegren, L., Kovalevsky, J., et al. 1997, A\&A, 323, L49 Pojmanski, G. 2002, AcA, 52, 397

Pollacco, D. L., Skillen, I., Collier Cameron, A., et al. 2006, PASP, 118, 1407

Popper, D. M. 1980, ARA\&A, 18, 115

Poveda, A., Herrera, M. A., Allen, C., Cordero, G., \& Lavalley, C. 1994, RMxAA, 28, 43

Pribulla, T. 2004, Spectroscopically and Spatially Resolving the Components of the Close Binary Stars, 318, 117

Prša, A., \& Zwitter, T. 2005, ApJ, 628, 426

Terrell, D., \& Wilson, R. E. 2005, Ap\&SS, 296, 221

Torres, C. A. O., Quast, G. R., da Silva, L., et al. 2006, A\&A, 460, 695

Wilson, R. E., \& Devinney, E. J. 1971, ApJ, 166, 605

Zasche, P. 2011, IBVS, 5991, 1 
P. Zasche et al.: GK Boo and AE For: Two low-mass eclipsing binaries with dwarf companions

Table 8. Heliocentric minima of GK Boo used for the analysis.

\begin{tabular}{|c|c|c|c|c|}
\hline$\overline{\text { HJD - } 2400000}$ & Error & Type & Filter & Reference \\
\hline 51260.8547 & 0.0007 & sec & - & IBVS 5060 \\
\hline 51273.9890 & & prim & - & A.Paschke - Rotse \\
\hline 51311.7377 & 0.0004 & prim & - & IBVS 5060 \\
\hline 53128.46588 & 0.00024 & $\mathrm{sec}$ & - & SWASP \\
\hline 53128.70498 & 0.00041 & prim & - & SWASP \\
\hline 53129.66141 & 0.00056 & prim & - & SWASP \\
\hline 53130.61697 & 0.00047 & prim & - & SWASP \\
\hline 53132.52777 & 0.00021 & prim & - & SWASP \\
\hline 53135.39435 & 0.00010 & prim & - & SWASP \\
\hline 53137.54360 & 0.00024 & $\mathrm{sec}$ & - & SWASP \\
\hline 53138.49891 & 0.00028 & $\mathrm{sec}$ & - & SWASP \\
\hline 53139.45491 & 0.00021 & $\mathrm{sec}$ & - & SWASP \\
\hline 53141.60560 & 0.00027 & prim & - & SWASP \\
\hline 53152.59424 & 0.00025 & prim & - & SWASP \\
\hline 53153.54982 & 0.00020 & prim & - & SWASP \\
\hline 53154.50553 & 0.00017 & prim & - & SWASP \\
\hline 53155.46071 & 0.00015 & prim & - & SWASP \\
\hline 53156.41642 & 0.00014 & prim & - & SWASP \\
\hline 53158.56492 & 0.00048 & $\mathrm{sec}$ & - & SWASP \\
\hline 53159.52074 & 0.00022 & $\mathrm{sec}$ & - & SWASP \\
\hline 53160.47640 & 0.00021 & $\mathrm{sec}$ & - & SWASP \\
\hline 53161.43169 & 0.00026 & $\mathrm{sec}$ & - & SWASP \\
\hline 53163.58313 & 0.00031 & prim & - & SWASP \\
\hline 53164.53862 & 0.00031 & prim & - & SWASP \\
\hline 53165.49392 & 0.00015 & prim & - & SWASP \\
\hline 53166.44965 & 0.00020 & prim & - & SWASP \\
\hline 53169.55361 & 0.00034 & $\mathrm{sec}$ & - & SWASP \\
\hline 53170.50939 & 0.00024 & $\mathrm{sec}$ & - & SWASP \\
\hline 53171.46489 & 0.00034 & $\mathrm{sec}$ & - & SWASP \\
\hline 53172.42077 & 0.00031 & $\mathrm{sec}$ & - & SWASP \\
\hline 53175.52707 & 0.00024 & prim & - & SWASP \\
\hline 53176.48289 & 0.00047 & prim & - & SWASP \\
\hline 53180.54262 & 0.00022 & $\mathrm{sec}$ & - & SWASP \\
\hline 53181.49835 & 0.00017 & $\mathrm{sec}$ & - & SWASP \\
\hline 53182.45376 & 0.00018 & $\mathrm{sec}$ & - & SWASP \\
\hline 53183.41009 & 0.00076 & $\mathrm{sec}$ & - & SWASP \\
\hline 53191.53267 & 0.00080 & $\mathrm{sec}$ & - & SWASP \\
\hline 53192.48678 & 0.00031 & $\mathrm{sec}$ & - & SWASP \\
\hline 53193.44381 & 0.00026 & $\mathrm{sec}$ & - & SWASP \\
\hline 53194.39850 & 0.00045 & $\mathrm{sec}$ & - & SWASP \\
\hline 53197.50383 & 0.00080 & prim & - & SWASP \\
\hline 53198.46046 & 0.00023 & prim & - & SWASP \\
\hline 53199.41623 & 0.00026 & prim & - & SWASP \\
\hline 53203.47632 & 0.00029 & $\mathrm{sec}$ & - & SWASP \\
\hline 53209.44918 & 0.00047 & prim & - & SWASP \\
\hline 53215.42110 & 0.00040 & $\mathrm{sec}$ & - & SWASP \\
\hline 53220.43588 & 0.00083 & prim & - & SWASP \\
\hline 53221.39317 & 0.00039 & prim & - & SWASP \\
\hline 53226.40796 & 0.00058 & $\mathrm{sec}$ & - & SWASP \\
\hline 53231.42630 & 0.00045 & prim & - & SWASP \\
\hline 53232.38275 & 0.00032 & prim & - & SWASP \\
\hline 53237.39732 & 0.00033 & sec & - & SWASP \\
\hline 53243.37030 & 0.00082 & prim & - & SWASP \\
\hline 53248.38731 & 0.00078 & $\mathrm{sec}$ & - & SWASP \\
\hline 53259.85637 & 0.00078 & $\mathrm{sec}$ & - & SWASP \\
\hline 53827.68574 & 0.00085 & prim & - & SWASP \\
\hline 53829.59740 & 0.00088 & prim & - & SWASP \\
\hline 53830.55201 & 0.00069 & prim & - & SWASP \\
\hline 53831.50729 & 0.00051 & prim & - & SWASP \\
\hline 53832.46270 & 0.00015 & prim & - & SWASP \\
\hline 53832.70138 & 0.00024 & $\mathrm{sec}$ & - & SWASP \\
\hline 53833.65681 & 0.00047 & $\mathrm{sec}$ & - & SWASP \\
\hline 53837.47803 & 0.00029 & $\mathrm{sec}$ & - & SWASP \\
\hline 53851.57305 & 0.00105 & prim & - & SWASP \\
\hline 53852.52690 & 0.00049 & prim & - & SWASP \\
\hline 53853.48213 & 0.00027 & prim & - & SWASP \\
\hline 53854.43799 & 0.00032 & prim & - & SWASP \\
\hline 53855.39312 & 0.00090 & prim & - & SWASP \\
\hline 53855.63171 & 0.00037 & $\mathrm{sec}$ & - & SWASP \\
\hline 53856.58695 & 0.00051 & $\mathrm{sec}$ & - & SWASP \\
\hline 53887.40789 & 0.00024 & prim & - & SWASP \\
\hline 53901.50285 & 0.00080 & $\mathrm{sec}$ & - & SWASP \\
\hline 54140.62657 & 0.00022 & prim & - & SWASP \\
\hline 54149.70458 & 0.00021 & prim & - & SWASP \\
\hline 54150.65998 & 0.00028 & prim & - & SWASP \\
\hline 54153.76487 & 0.00119 & $\mathrm{sec}$ & - & SWASP \\
\hline 54154.71989 & 0.00026 & $\mathrm{sec}$ & - & SWASP \\
\hline 54155.67581 & 0.00061 & $\mathrm{sec}$ & - & SWASP \\
\hline 54156.63161 & 0.00053 & $\mathrm{sec}$ & - & SWASP \\
\hline 54157.58667 & 0.00065 & $\mathrm{sec}$ & - & SWASP \\
\hline 54159.73799 & 0.00019 & prim & - & SWASP \\
\hline 54160.69347 & 0.00018 & prim & - & SWASP \\
\hline 54161.64928 & 0.00022 & prim & - & SWASP \\
\hline
\end{tabular}

Table 8. continued.

\begin{tabular}{|c|c|c|c|c|}
\hline$\overline{\text { HJD - } 2400000}$ & Error & Type & Filter & Reference \\
\hline 54162.60325 & 0.00019 & prim & - & SWASP \\
\hline 54163.55942 & 0.00065 & prim & - & SWASP \\
\hline 54165.70909 & 0.00037 & sec & - & SWASP \\
\hline 54166.66462 & 0.00022 & $\mathrm{sec}$ & - & SWASP \\
\hline 54167.62053 & 0.00034 & $\mathrm{sec}$ & - & SWASP \\
\hline 54170.72638 & 0.00023 & prim & - & SWASP \\
\hline 54171.68178 & 0.00043 & prim & - & SWASP \\
\hline 54189.59755 & 0.00032 & sec & - & SWASP \\
\hline 54190.55322 & 0.00019 & $\mathrm{sec}$ & - & SWASP \\
\hline 54191.50702 & 0.00053 & $\mathrm{sec}$ & - & SWASP \\
\hline 54194.61418 & 0.00029 & prim & - & SWASP \\
\hline 54195.57050 & 0.00029 & prim & - & SWASP \\
\hline 54202.49743 & 0.00046 & $\mathrm{sec}$ & - & SWASP \\
\hline 54206.55909 & 0.00039 & prim & - & SWASP \\
\hline 54208.46922 & 0.00027 & prim & - & SWASP \\
\hline 54208.70823 & 0.00143 & $\mathrm{sec}$ & - & SWASP \\
\hline 54210.61908 & 0.00030 & $\mathrm{sec}$ & - & SWASP \\
\hline 54212.53056 & 0.00050 & $\mathrm{sec}$ & - & SWASP \\
\hline 54213.48698 & 0.00049 & $\mathrm{sec}$ & - & SWASP \\
\hline 54214.44267 & 0.00029 & $\mathrm{sec}$ & - & SWASP \\
\hline 54214.68147 & 0.00039 & prim & - & SWASP \\
\hline 54215.63620 & 0.00057 & prim & - & SWASP \\
\hline 54216.59233 & 0.00019 & prim & - & SWASP \\
\hline 54217.54805 & 0.00038 & prim & - & SWASP \\
\hline 54218.50359 & 0.00021 & prim & - & SWASP \\
\hline 54219.45928 & 0.00030 & prim & - & SWASP \\
\hline 54220.41494 & 0.00069 & prim & BVR & PS \\
\hline 54220.41498 & 0.00029 & prim & - & SWASP \\
\hline 54222.56423 & 0.00066 & sec & BVR & PS \\
\hline 54222.56444 & 0.00028 & $\mathrm{sec}$ & - & SWASP \\
\hline 54223.51949 & 0.00169 & $\mathrm{sec}$ & BVR & PS \\
\hline 54223.51994 & 0.00108 & $\mathrm{sec}$ & - & SWASP \\
\hline 54224.47565 & 0.00035 & $\mathrm{sec}$ & - & SWASP \\
\hline 54225.43110 & 0.00022 & $\mathrm{sec}$ & - & SWASP \\
\hline 54225.67010 & 0.00026 & prim & - & SWASP \\
\hline 54226.38671 & 0.00034 & sec & - & SWASP \\
\hline 54226.62544 & 0.00036 & prim & - & SWASP \\
\hline 54227.58111 & 0.00026 & prim & - & SWASP \\
\hline 54228.53676 & 0.00031 & prim & - & SWASP \\
\hline 54230.44800 & 0.00019 & prim & - & SWASP \\
\hline 54231.40365 & 0.00019 & prim & - & SWASP \\
\hline 54231.64168 & 0.00048 & $\mathrm{sec}$ & - & SWASP \\
\hline 54232.59790 & 0.00032 & $\mathrm{sec}$ & - & SWASP \\
\hline 54233.55304 & 0.00025 & $\mathrm{sec}$ & - & SWASP \\
\hline 54234.50899 & 0.00034 & $\mathrm{sec}$ & - & SWASP \\
\hline 54235.46550 & 0.00054 & $\mathrm{sec}$ & - & SWASP \\
\hline 54236.42047 & 0.00028 & $\mathrm{sec}$ & - & SWASP \\
\hline 54236.65842 & 0.00085 & prim & - & SWASP \\
\hline 54237.37517 & 0.00137 & sec & BVR & PS \\
\hline 54239.52553 & 0.00091 & prim & BVR & PS \\
\hline 54249.55879 & 0.00027 & prim & - & SWASP \\
\hline 54250.51471 & 0.00031 & prim & - & SWASP \\
\hline 54251.47023 & 0.00057 & prim & - & SWASP \\
\hline 54252.42644 & 0.00028 & prim & - & SWASP \\
\hline 54254.57647 & 0.00059 & sec & - & SWASP \\
\hline 54256.48657 & 0.00172 & $\mathrm{sec}$ & BVR & PS \\
\hline 54256.48655 & 0.00039 & $\mathrm{sec}$ & - & SWASP \\
\hline 54257.44222 & 0.00025 & $\mathrm{sec}$ & - & SWASP \\
\hline 54261.50367 & 0.00031 & prim & - & SWASP \\
\hline 54262.45860 & 0.00020 & prim & - & SWASP \\
\hline 54263.41487 & 0.00037 & prim & - & SWASP \\
\hline 54265.56380 & 0.00079 & sec & - & SWASP \\
\hline 54266.51892 & 0.00042 & $\mathrm{sec}$ & - & SWASP \\
\hline 54267.47491 & 0.00020 & $\mathrm{sec}$ & - & SWASP \\
\hline 54268.43084 & 0.00017 & $\mathrm{sec}$ & - & SWASP \\
\hline 54271.53663 & 0.00070 & prim & - & SWASP \\
\hline 54272.49225 & 0.00033 & prim & - & SWASP \\
\hline 54273.44808 & 0.00026 & prim & - & SWASP \\
\hline 54276.55314 & 0.00095 & $\mathrm{sec}$ & - & SWASP \\
\hline 54277.50813 & 0.00065 & $\mathrm{sec}$ & - & SWASP \\
\hline 54278.46353 & 0.00031 & $\mathrm{sec}$ & - & SWASP \\
\hline 54279.41942 & 0.00021 & $\mathrm{sec}$ & - & SWASP \\
\hline 54305.45758 & 0.00157 & prim & BVR & PS \\
\hline 54307.37045 & 0.00141 & prim & BVR & PS \\
\hline 54568.70649 & 0.00072 & prim & - & Piofthesky \\
\hline 54568.94557 & 0.00097 & $\mathrm{sec}$ & - & Piofthesky \\
\hline 54925.36514 & 0.0001 & $\mathrm{sec}$ & $\mathrm{R}$ & OEJV 107 \\
\hline 54937.3112 & 0.0020 & $\mathrm{sec}$ & $\mathrm{Ir}$ & IBVS 5918 \\
\hline 54937.5462 & 0.0005 & prim & $\mathrm{Ir}$ & IBVS 5918 \\
\hline 54947.34260 & 0.0014 & $\mathrm{sec}$ & $\mathrm{R}$ & OEJV 107 \\
\hline 54958.8085 & 0.0007 & $\mathrm{sec}$ & $\mathrm{V}$ & IBVS 5894 \\
\hline 54959.52489 & 0.0001 & prim & $\mathrm{R}$ & OEJV 107 \\
\hline 55354.40380 & 0.00148 & sec & BVR & PS \\
\hline
\end{tabular}


A\&A 537, A109 (2012)

Table 8. continued.

\begin{tabular}{lllll}
\hline \hline HJD - 2400000 & Error & Type & Filter & Reference \\
\hline 55364.4367 & 0.0001 & sec & BVRI & IBVS 5965 \\
55385.45891 & 0.00131 & sec & BVR & PS \\
55386.41381 & 0.00104 & sec & BVR & PS \\
55391.43075 & 0.00097 & prim & BVR & PS \\
55392.38658 & 0.00113 & prim & BVR & PS \\
55590.66335 & 0.00036 & prim & - & RU \\
55599.50146 & 0.00018 & sec & - & RU \\
55616.46275 & 0.00005 & prim & B & PZ \\
55616.46266 & 0.00012 & prim & R & RU \\
55619.56796 & 0.00010 & sec & R & RU \\
55634.61898 & 0.00016 & prim & R & RU \\
55640.58947 & 0.00019 & sec & R & RU \\
55644.41169 & 0.00005 & sec & B & PZ \\
55650.62303 & 0.00014 & sec & R & RU \\
55651.34031 & 0.00113 & prim & B & PZ \\
55662.56706 & 0.00013 & sec & R & RU \\
55671.40664 & 0.00007 & prim & VR & PZ \\
55685.50021 & 0.00012 & sec & R & RU \\
55687.41150 & 0.00011 & sec & BVR & PZ \\
55692.42853 & 0.00004 & prim & BVR & PZ \\
55700.55071 & 0.00004 & prim & BVR & PZ \\
55707.47805 & 0.00008 & sec & BVR & PZ \\
\hline
\end{tabular}

Table 9. Heliocentric minima of AE For used for the analysis.

\begin{tabular}{|c|c|c|c|c|}
\hline HJD - 2400000 & Error & Type & Filter & Reference \\
\hline 48500.6581 & 0.001 & prim & $\mathrm{Hp}$ & Hipparcos \\
\hline 51180.9089 & & prim & $\mathrm{R}$ & VSOLJ 37 \\
\hline 51180.9090 & 0.0002 & prim & $\mathrm{R}$ & VSOLJ 47 \\
\hline 51191.0099 & & prim & $\mathrm{R}$ & VSOLJ 37 \\
\hline 51191.0100 & 0.0002 & prim & $\mathrm{R}$ & VSOLJ 47 \\
\hline 51191.9279 & & prim & V & VSOLJ 37 \\
\hline 51191.9280 & 0.0001 & prim & V & VSOLJ 47 \\
\hline 51196.9770 & 0.0001 & $\mathrm{sec}$ & $\mathrm{R}$ & VSOLJ 47 \\
\hline 51196.9774 & & $\mathrm{sec}$ & $\mathrm{R}$ & VSOLJ 37 \\
\hline 51504.11886 & & prim & I & VSOLJ 37 \\
\hline 51504.1190 & 0.0002 & prim & I & VSOLJ 47 \\
\hline 52065.14178 & 0.00121 & prim & V & ASAS \\
\hline 52065.60355 & 0.0025 & $\mathrm{sec}$ & V & ASAS \\
\hline 52235.0140 & & prim & I & VSOLJ 39 \\
\hline 52240.9825 & & $\mathrm{sec}$ & I & VSOLJ 39 \\
\hline 52258.8860 & 0.0002 & prim & $\mathrm{R}$ & VSOLJ 39 \\
\hline 52605.9710 & & prim & I & VSOLJ 40 \\
\hline 52818.07823 & 0.00077 & prim & $\mathrm{V}$ & ASAS \\
\hline 52818.53514 & 0.00165 & sec & $\mathrm{V}$ & ASAS \\
\hline 52901.1754 & & $\mathrm{sec}$ & I & VSOLJ 42 \\
\hline 52929.1801 & & prim & I & VSOLJ 42 \\
\hline 52936.0674 & & $\mathrm{sec}$ & I & VSOLJ 42 \\
\hline 52957.1865 & & $\mathrm{sec}$ & V & VSOLJ 42 \\
\hline 52970.0410 & & $\mathrm{sec}$ & I & VSOLJ 42 \\
\hline 52976.0097 & & prim & I & VSOLJ 42 \\
\hline 52987.0267 & & prim & V & VSOLJ 42 \\
\hline 52987.9466 & & prim & I & VSOLJ 42 \\
\hline 53300.1379 & & prim & V & VSOLJ 43 \\
\hline 53335.0300 & & prim & V & VSOLJ 43 \\
\hline 53340.0796 & & sec & V & VSOLJ 43 \\
\hline 53705.0677 & 0.0002 & prim & $\mathrm{V}$ & VSOLJ 44 \\
\hline 53728.02219 & 0.00093 & prim & $\mathrm{V}$ & ASAS \\
\hline 53728.48316 & 0.00194 & $\mathrm{sec}$ & V & ASAS \\
\hline 54039.75501 & 0.00027 & $\mathrm{sec}$ & - & PiOfTheSky \\
\hline 54040.67301 & 0.00045 & $\mathrm{sec}$ & - & PiOfTheSky \\
\hline 54047.1000 & 0.0001 & $\mathrm{sec}$ & $\mathrm{V}$ & VSOLJ $45^{\circ}$ \\
\hline 54052.60840 & 0.00149 & $\mathrm{sec}$ & - & PiOfTheSky \\
\hline 54448.81542 & 0.00039 & prim & - & PiOfTheSky \\
\hline 54726.11607 & 0.00187 & prim & $\mathrm{V}$ & ASAS \\
\hline 54726.57421 & 0.00131 & sec & $\mathrm{V}$ & ASAS \\
\hline 54862.9297 & 0.0002 & prim & $\mathrm{V}$ & VSOLJ 50 \\
\hline 55558.0139 & & prim & $\mathrm{V}$ & VSOLJ 51 \\
\hline 55570.41065 & 0.00072 & $\mathrm{sec}$ & BVR & PZ - SAAO \\
\hline 55571.32812 & 0.00105 & $\mathrm{sec}$ & BVR & PZ - SAAO \\
\hline 55576.37847 & 0.00065 & prim & BVR & PZ - SAAO \\
\hline
\end{tabular}

